УДК 541.138 .3

DOI 10.17072/2223-1838-2021-4-263-270

В.В. Третьякова, А.Е. Пономарева, В.В. Пантелеева, А.Б. Шеин

Пермский государственный национальный исследовательский университет, Пермь, Россия

\title{
СОСТАВ, СТРУКТУРА И ЭЛЕКТРОХИМИЧЕСКАЯ АКТИВНОСТЬ СИЛИЦИДА ТИТАНА В РЕАКЦИИ ВЫДЕЛЕНИЯ ВОДОРОДА
}

\begin{abstract}
Методами микрорентгеноспектрального и рентгеноструктурного анализов исследованы фазовый состав и структура силицида титана. Установлено, что исследованный силицид представляет собой однофазную систему, состоящую из высокотемпературной модификаиии $\mathrm{TiSi}_{2}$ с ромбической гранецентрированной решеткой. Методами поляризачионных и емкостных измерений изучено катодное поведение $\mathrm{TiSi}_{2}$-электрода. Обнаружено, что катодные потенииостатические кривые силицида в растворах 0,5 $\mathrm{M} \mathrm{H}_{2} \mathrm{SO}_{4}$; 0,5 $\mathrm{M} \mathrm{H}_{2} \mathrm{SO}_{4}+0,005 \mathrm{M} \mathrm{NaF} \mathrm{ul,0} \mathrm{M}$ $\mathrm{NaOH} \mathrm{имеют} \mathrm{тафелевские} \mathrm{участки} \mathrm{с} \mathrm{наклонами} \mathrm{0,120;} \mathrm{0,097} \mathrm{и} \mathrm{0,109} \mathrm{В} \mathrm{и} \mathrm{характеризуются} \mathrm{ве-}$ личиной перенапряжения выделения водорода 0,90; 0,64 и 0,74 В (nри $\left.i=1 \mathrm{~A} / \mathrm{cm}^{2}\right)$ соответственно. В растворе серной кислоты дисилици титана относится к материалам с высоким перенапряжением выделения водорода, во фторидсодержащем сернокислом растворе и в щелочном растворе - к материалам с невысоким перенапряжением выделения водорода. На основании измерений дифференциальной емкости TiSi $i_{2}$-электрода (nри $f=10 \kappa Г$ и) сделано заключение о присутствии на поверхности силицида в кислом бесфторидном электролите тонкой пленки диоксида кремния $\left(\mathrm{Si}+2 \mathrm{H}_{2} \mathrm{O} \rightarrow \mathrm{SiO}_{2}+4 \mathrm{H}^{+}+4 e^{-}\right)$.
\end{abstract}

Ключевые слова: силицид титана; реакция выделения водорода; сернокислый электролит; щелочной электролит

Поступила в редакцฺию 15.10.2021; после доработки 12.11.2021; принята к публикации 24.11.2021

\section{V.V. Tretyakova, A.E. Ponomareva, V.V. Panteleeva, A.B. Shein}

Perm State University, Perm, Russia

\section{COMPOSITION, STRUCTURE AND ELECTROCHEMICAL ACTIVITY OF TITANIUM SILICIDE IN HYDROGEN EVOLUTION REACTION}

The phase composition and structure of titanium silicide have been investigated by $X$-ray diffraction and X-ray spectral microanalysis methods. It has been found that the investigated silicide is a singlephase system consisting of a high-temperature $\mathrm{TiSi}_{2}$ modification with a rhombic face-centered lattice. The cathodic behavior of the TiSi $i_{2}$ electrode has been studied by the methods of polarization and capacitance measurements. It has been found that the cathodic potentiostatic curves of silicide in solutions of 0,5 $\mathrm{M} \mathrm{H}_{2} \mathrm{SO}_{4} ; 0,5 \mathrm{M} \mathrm{H}_{2} \mathrm{SO}_{4}+0,005 \mathrm{M} \mathrm{NaF}$ andl,0 $\mathrm{M}$ NaOHhave Tafel sections with slopes of 0,120;0,097 and 0,109 $\mathrm{V}$ and they are characterized by the values of the hydrogen evolution overvoltage 0,90; 0,64 and $0,74 \mathrm{~V}\left(\right.$ at $\left.i=1 \mathrm{~A} / \mathrm{cm}^{2}\right)$, respectively. Titanium disilicide in sulfuric acid solution belongs to materials with a high overvoltage of hydrogen evolution, but in a fluoride-containing sulfuric acid solution and in an alkaline solution - to materials with a low overvoltage of hydrogen evolution. Based on measurements of the differential capacitance of the TiSi $i_{2}$ electrode (at $f=10 \mathrm{kHz}$ ), it has been concluded that a thin silicon dioxide film $\left(\mathrm{Si}+2 \mathrm{H}_{2} \mathrm{O} \rightarrow \mathrm{SiO}_{2}+4 \mathrm{H}^{+}+4 e^{-}\right)$is present on the surface of the silicide in the acidic fluoride-free electrolyte.

Keywords: titanium silicide; hydrogen evolution reaction; sulfuric acid solution;alkaline electrolyte

Received 15.10.2021; revised 12.11.2021; accepted 24.11.2021

(СТретьякова В.В., Пономарева А.Е., Пантелеева В.В., Шеин А.Б., 2021 
Катодная реакция выделения водорода (р.в.в.) - одна из важнейших электрохимических реакций. В настоящее время в связи с интенсивным развитием водородной энергетики значительное внимание уделяется поиску материалов, активных в реакции электролитического выделения водорода, и разработке способов повышения их эффективности при получении водорода [1]. Перспективными в этом отношении материалами являются металлоподобные соединения, например карбиды, нитриды, силициды, германиды переходных металлов, тройные соединения [2-11]. Изучению кинетических закономерностей р.в.в. на силицидах переходных металлов посвящен ряд работ [2-4, 7-10]; для некоторых силицидов отмечены более низкие перенапряжения выделения водорода по сравнению с соответствующими чистыми металлами.

Предметом настоящей работы является изучение фазового состава и структуры силицида титана, полученного методом Бриджмена, и определение его электрохимической активности в реакции выделения водорода в сернокислом и щелочном электролитах.

\section{Материалы и методика эксперимента}

Материалом для исследования служил силицид титана, который был получен методом Бриджмена. Микроструктуру, химический состав силицида титана, морфологию рабочей поверхности электрода исследовали с помощью металлографического микроскопа «Olimpus BX51» с системой визуализации, сканирующего электронного микроскопа S3400N фирмы Hitachi (Япония) с приставкой для энергодисперсионного анализа Quantax 200 фирмы Bruker (Германия). Структурная аттестация образца осуществлена методом рентгеновской дифракции на порошковомдифрактометре «D8 Advance» с системой визуализации.

Электрохимические измерения проведены при температуре $25{ }^{\circ} \mathrm{C}$ в условиях естественной аэрации в неперемешиваемых растворах 0,5 моль/л $\mathrm{H}_{2} \mathrm{SO}_{4} ; \quad 0,5$ моль/л $\mathrm{H}_{2} \mathrm{SO}_{4}+$ 0,005 моль/л $\mathrm{NaF}$ и 1,0 моль/л $\mathrm{NaOH}$. Для приготовления растворов использовали деионизованную воду (удельное сопротивление воды 18,2 МОм·см, содержание органического углерода - 4 мкг/л), полученную с помощью системы очистки воды Milli-Q фирмы Millipore (Франция), и реактивы $\mathrm{H}_{2} \mathrm{SO}_{4}, \mathrm{NaF}, \mathrm{NaOH}$ квалификации «х.ч.». Измерения проведены с помощью потенциостата-гальваностата с встроенным частотным анализатором Solartron 1280C фирмы Solartron Analytical (Великобритания) в электрохимической ячейке ЯСЭ-2 с разделенными пористой стеклянной диафрагмой катодным и анодным отделениями. В качестве электрода сравнения использовали насыщенный хлоридсеребряный электрод, в качестве вспомогательного электрода - платиновый электрод. Потенциалы в работе приведены относительно стандартного водородного электрода.

Перед проведением измерений рабочую поверхность электрода шлифовали абразивными бумагами с последовательным уменьшением размера зерна, обезжиривали этиловым спиртом, ополаскивали рабочим раствором. После погружения в раствор электрод подвергали катодной поляризации при плотности тока 0,5 мA/ $\mathrm{cm}^{2}$ в течение 10 мин, затем выдерживали при потенциале разомкнутой цепи до установления стационарного значения, далее регистрировали катодные потенциостатические 
кривые в направлении от высоких к низким потенциалам с шагом 0,03 B.

При электрохимических измерениях и обработке полученных данных использовали программы CorrWare2, CView2 (Scribner Associates, Inc.).

\section{Результаты и их обсуждение}

Методами оптической микроскопии, сканирующей электронной микроскопии и микрорентгеноспектрального анализа исследованы микроструктура и элементный состав силицида титана (рис. 1). Микрорентгеноспектральный анализ показал, что распределение элементов $\mathrm{Ti}$ и $\mathrm{Si}$ в образце является равномер- ным, соотношение элементов $(32,6 \pm 1,6$ ат. \% титана; 67,4 $\pm 2,4$ ат. \% кремния) соответствует соединению $\mathrm{TiSi}_{2}$.

Дисилицид титана имеет две полиморфные модификации [12]. Низкотемпературная метастабильная модификация (C49) имеет ромбическую базоцентрированную решетку, пространственная группа $\mathrm{Cmcm}$, периоды решетки: $a=0,362 \mathrm{нM}, b=1,376 \mathrm{нM}, c=0,360$ нм. Высокотемпературная модификация (C54) является стабильной и имеет ромбическую гранецентрированную решетку, пространственная группа $F d d d$, периоды решетки: $a=0,8279$ нм, $b=0,4819$ нм, $c=0,8568$ нм.

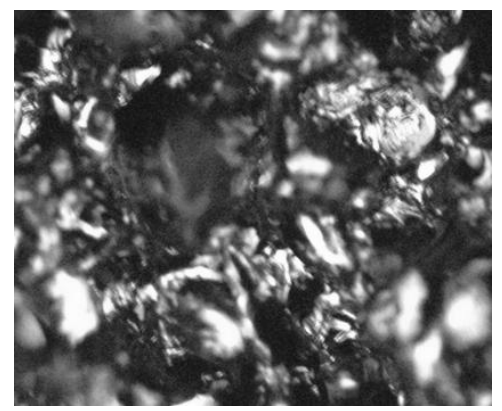

a

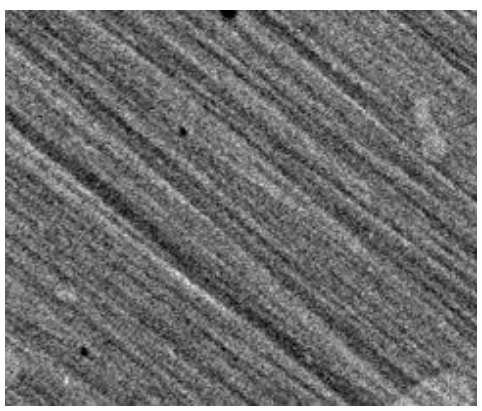

б

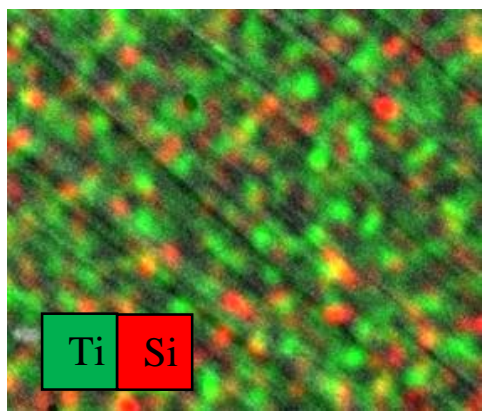

B

Рис. 1. Микрофотографии поверхности силицида титана: а - исходный образец (×100);

б - механическая зачистка $(\times 1000)$; в - распределение элементов по поверхности

Из рентгенографического анализа следует, что исследуемый образец представляет собой однофазную систему, состоящую из высокотемпературной модификации $\mathrm{TiSi}_{2}$ с ромбической гранецентрированной решеткой (рис. 2).

Катодные потенциостатические кривые $\mathrm{TiSi}_{2}$-электрода в растворах 0,5 моль/л $\mathrm{H}_{2} \mathrm{SO}_{4}$; 0,5 моль/л $\mathrm{H}_{2} \mathrm{SO}_{4}+0,005$ моль/лNaF и 1,0 моль/л $\mathrm{NaOH}$ приведены на рис. 3. На катодной кривой силицида в 0,5 моль/л $\mathrm{H}_{2} \mathrm{SO}_{4}$ отмечается наличие линейного участка с наклоном 0,120 В в области перенапряжений от 0,28 до -0,46 В; величина перенапряжения вы- деления водорода при $i=1 \mathrm{~A} / \mathrm{cm}^{2}$ составляет 0,90 B.

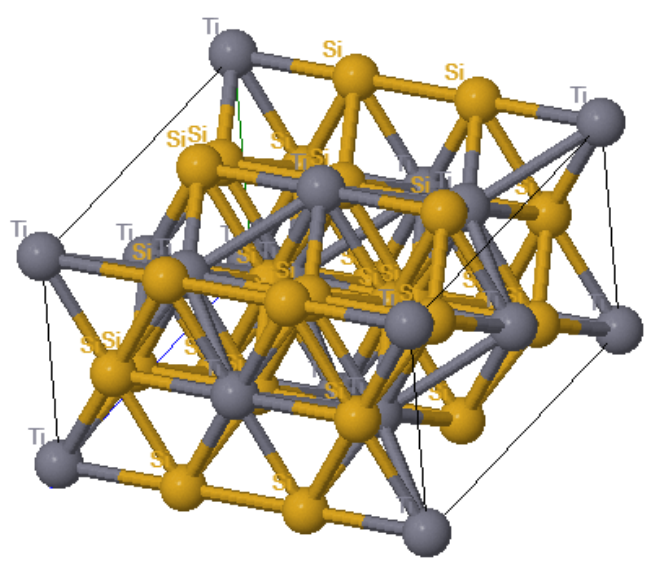

Рис. 2. Кристаллическая структура высокотемпературной модификации $\mathrm{TiSi}_{2}$ 
В растворе 0,5 моль/л $\mathrm{H}_{2} \mathrm{SO}_{4}$, содержащем 0,005 моль/л $\mathrm{NaF}$, диапазон перенапряжений регистрации линейной зависимости $\eta$ от $\lg i$ по сравнению с бесфторидным раствором уменьшается; отмечается снижение наклона поляризационной кривой и величины $\eta$ при $i=1$ $\mathrm{A} / \mathrm{cm}^{2}$ (см. таблицу).

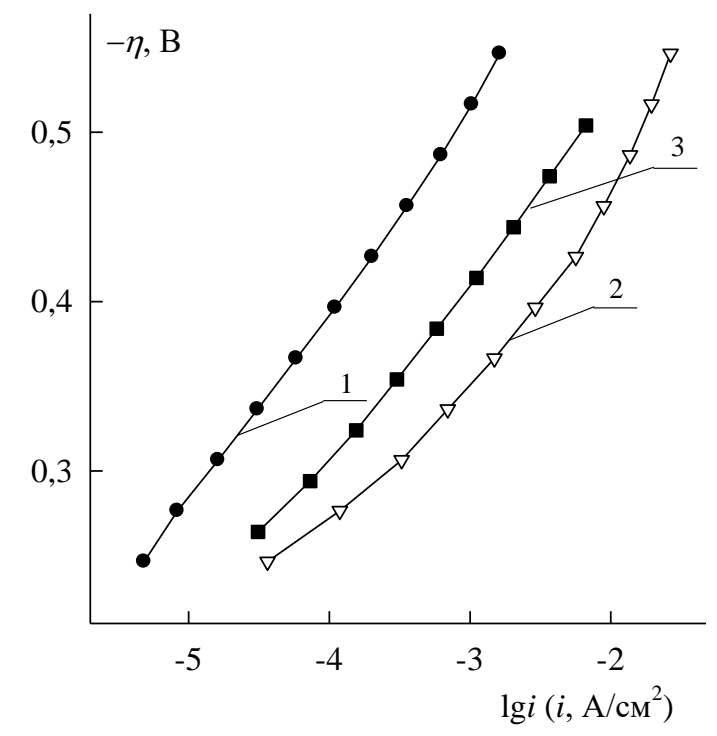

Рис. 3. Катодные потенциостатические кривые $\mathrm{TiSi}_{2}$-электрода в растворах: 1 - 0,5 моль/л $\mathrm{H}_{2} \mathrm{SO}_{4}$;

2 - 0,5 моль/л $\mathrm{H}_{2} \mathrm{SO}_{4}+0,005$ моль/л $\mathrm{NaF}$; 3 - 1,0 моль/л $\mathrm{NaOH}$

В щелочном электролите катодная кривая $\mathrm{TiSi}_{2}$-электрода имеет тафелевский участок с наклоном 0,109 Вв области $\eta$ от $-0,29$ до $-0,50$ В и характеризуется величиной перенапряжения 0,74 В. В бесфторидном растворе серной кислоты дисилицид титана относится к материалам с высоким перенапряжением выделения водорода, во фторидсодержащем сернокислом растворе и в щелочном растворе - к материалам со средним перенапряжением выделения водорода.Дифференциальная емкость $\mathrm{TiSi}_{2}$-электрода, измеренная при частоте переменного тока $f=10$ кГц, в растворе $0,5 \mathrm{M}$ $\mathrm{H}_{2} \mathrm{SO}_{4}$ в исследованной области составляет

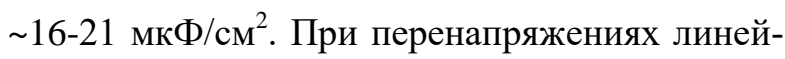
ного участка на катодных кривых регистрируется слабая зависимость емкости от $\eta$ (рис. 4).

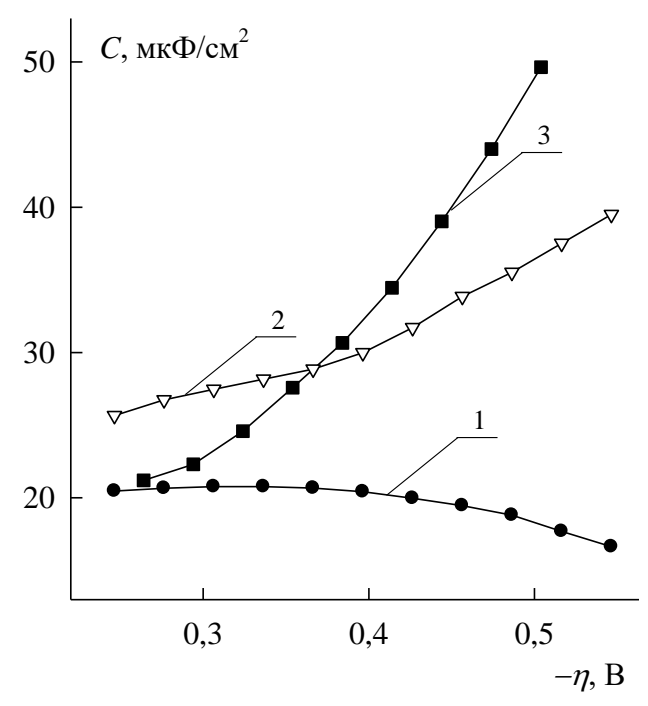

Рис. 4. Зависимость дифференциальной емкости $\mathrm{TiSi}_{2}$-электрода при частоте 10 кГц от перенапряжения в растворах: $1-0,5$ моль/л $\mathrm{H}_{2} \mathrm{SO}_{4}$;

2 - 0,5 моль/л $\mathrm{H}_{2} \mathrm{SO}_{4}+0,005$ моль/л $\mathrm{NaF}$;

$$
3-1,0 \text { моль/л } \mathrm{NaOH}
$$

Кинетические параметры реакции выделения водорода на $\mathrm{TiSi}_{2}$-электроде

\begin{tabular}{|c|c|c|c|}
\hline Раствор & $-\eta, \mathrm{B}$ & $-\left(\frac{\partial \eta}{\partial \lg i}\right)_{c}, \mathrm{~B}$ & $\begin{array}{c}-\eta, \mathrm{B} \\
\text { при } i=1 \mathrm{~A} / \mathrm{cm}^{2}\end{array}$ \\
\hline $0,5 \mathrm{M} \mathrm{H}_{2} \mathrm{SO}_{4}$ & $0,28-0,46$ & 0,120 & 0,90 \\
$0,5 \mathrm{M} \mathrm{H}_{2} \mathrm{SO}_{4}+0,005 \mathrm{M} \mathrm{NaF}$ & $0,31-0,43$ & 0,097 & 0,64 \\
$1,0 \mathrm{M} \mathrm{NaOH}$ & $0,29-0,50$ & 0,109 & 0,74 \\
\hline
\end{tabular}

Низкие значения емкости силицида в растворе серной кислоты, по-видимому, обусловлены присутствием на его поверхности тонкой диэлектрической пленки диоксида кремния
$\left(\mathrm{Si}+2 \mathrm{H}_{2} \mathrm{O} \rightarrow \mathrm{SiO}_{2}+4 \mathrm{H}^{+}+4 \mathrm{e}^{-}, E^{0}=-0,86 \mathrm{~B}\right.$

[13]). Согласно [14], дисилицид титана (высокотемпературная модификация $\mathrm{TiSi}_{2}$ с ромбической гранецентрированной решеткой, про- 
странственная группа $F d d d$ ) при комнатной температуре относится к металлическим проводникам и характеризуется удельным сопротивлением, не превышающим значений 20 мкОм·см. Оксидная пленка формируется на силициде уже при потенциале коррозии и не подвергается восстановлению во время катодной поляризации. Авторами [2] также показано, что $\mathrm{SiO}_{2}$ является устойчивым в кислых средах при невысоких катодных поляризациях.

Введение в раствор 0,5 моль/л $\mathrm{H}_{2} \mathrm{SO}_{4}$ фторида натрия, вызывающего растворение диоксида кремния [15], приводит к увеличению дифференциальной емкости силицида в 1,32,1 раза (рис. 4). Заметное повышение емкости в присутствии фторида (наряду с растворением оксида) также может быть связано с развитием поверхности. В щелочном растворе емкость $\mathrm{TiSi}_{2}$-электрода (при сопоставимых с кислым электролитом величинах перенапряжения)

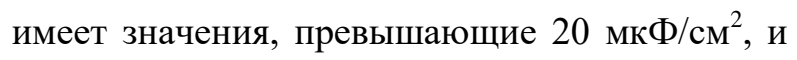
увеличивается с ростом потенциала. Более высокие значения дифференциальной емкости силицида в присутствии фторида и в щелочном электролите подтверждают предположение о том, что низкие значения емкости и, повидимому, высокое перенапряжение выделения водорода на $\mathrm{TiSi}_{2}$ в кислом бесфторидном растворе обусловлены присутствием на его поверхности тонкой оксидной пленки.

\section{Заключение}

Установлено, что исследованный силицид титана представляет собой однофазную систему, состоящую из высокотемпературной модификации $\mathrm{TiSi}_{2}$ с ромбической гранецентрированной решеткой. Катодные потенциостатические кривые $\mathrm{TiSi}_{2}$-электрода в растворах 0,5 моль/л $\mathrm{H}_{2} \mathrm{SO}_{4} ; 0,5$ моль/л $\mathrm{H}_{2} \mathrm{SO}_{4}+0,005$ моль/л $\mathrm{NaF}$ и 1,0 моль/л $\mathrm{NaOH}$ имеют тафелевские участки с наклонами 0,$120 ; 0,097$ и 0,109 В и характеризуются величиной перенапряжения выделения водорода при $\mathrm{i}=1 \mathrm{~A} / \mathrm{cm}^{2}$, равной 0,$90 ; 0,64$ и 0,74 В соответственно. В бесфторидном растворе серной кислоты дисилицид титана относится к материалам с высоким перенапряжением выделения водорода, которое обусловлено присутствием на его поверхности тонкой оксидной пленки; во фторидсодержащем сернокислом растворе и в щелочном растворе - к материалам с невысоким перенапряжением выделения водорода. Последнее определяет перспективность применения силицида в качестве электрокатализатора при получении водорода.

\section{Конфликт интересов}

Авторы заявляют об отсутствии конфликта интересов.

\section{Список литературы}

1. Козин Л.Ф., Волков С.В. Водородная энергетика и экология. Киев: Наукова думка, 2002. $336 \mathrm{c}$.

2. Shamsul Huq A.K.M., Rosenberg A.J.J. Electrochemical Behavior of Nickel Compounds: I. The Hydrogen Evolution Reaction on NiSi, $\mathrm{NiAs}, \mathrm{NiSb}, \mathrm{NiS}, \mathrm{NiTe}_{2}$, and Their Constituent Elements // Journal of the Electrochemical Society. 1964. V. 111, № 3. P. 270-278.

3. Vijh A.K., Belanger G., Jacques R. Electrochemical activity of silicides of some transition metals for the hydrogen evolution reaction in acidic solutions // International Journal of Hydrogen Energy. 1990. V. 15, № 11. P. 789-794.

4. Vijh A.K., Belanger G., Jacques R. Electrolysis of water on silicides of some transition metals in alkaline solutions // International Journal of Hydrogen Energy. 1992. V. 15, № 7. P. 479. 
5. Shein A.B., Kichigin V.I., Konyk M., Romaka L., Stadnyk Yu. Study of the kinetics and mechanism of the hydrogen evolution reaction on $\mathrm{CeMe}_{2} \mathrm{Ge}_{2}$ electrodes $(\mathrm{Me}=\mathrm{Fe}, \mathrm{Co}, \mathrm{Ni}) / /$ Chemistry of Metals and Alloys. 2013. Vol. 6, № 3-4. P. 113-120.

6. Meyer S., Nikiforov A.V., Petrushina I.M., Kohler K., Christensen E., Jensen J.O., Bjerrum N.J. Transition metal carbides (WC, $\left.\mathrm{Mo}_{2} \mathrm{C}, \mathrm{TaC}, \mathrm{NbC}\right)$ as potential electrocatalysts for the hydrogen evolution reaction (HER) at medium temperatures // International Journal of Hydrogen Energy. 2015. Vol. 40, № 7. P. 2905-2911.

7. Safizadeh F., Ghali E., Houlachi G.Electrocatalysis developments for hydrogen evolution reaction in alkaline solutions - A Review // International Journal of Hydrogen Energy. 2015. Vol. 40, № 1. P. 256-274.

8. Kichigin V.I., Shein A.B. Kinetics and mechanism of hydrogen evolution reaction on cobalt silicides in alkaline solutions // ElectrochimicaActa. 2015. Vol. 164. P. 260-266.

9. Кузьминых М.М., Пантелеева В.В., Шеин А.Б. Катодное выделение водорода на дисилициде железа. I. Щелочная среда // Известия вузов. Химия и химическая технология. 2019. Вып. 62, № 1. Р. 38-45.

10. Кузьминых М.М., Пантелеева В.В., Шеин А.Б. Катодное выделение водорода на дисилициде железа. II. Кислая среда. // Известия вузов. Химия и химическая технология. 2019. Вып. 62,№ 2. Р. 59-64.

11. Theerthagiri J., Lee S.J., Murthy A.P., Madhavan J., Choi M.Y. Fundamental aspects and recent advances in transition metal nitrides as electrocatalysts for hydrogen evolution reaction: A review // Current Opinion in Solid
State and Materials Science. 2020. Vol. 24, № 1.P. $100-805$.

12. Гельд П.В., Сидоренко Ф.А. Силициды переходных металлов четвертого периода. М.: Металлургия, 1981. 632 с.

13. Справочник по электрохимии /под ред. А.М.Сухотина. Л.: Химия, 1981.488 с.

14. Мьюрарка М.Силициды для СБИС. М.: Мир, 1986. 176 с.

15. Zhang X.G. Electrochemistry of silicon and its oxide.New York, Kluwer Academic/Plenum Publ., 2001, 510 p.

\section{References}

1. Kozin, L.F. and Volkov, S.V. (2002) Vodorodnaya energetika i ekologiya [Hydrogen energy and ecology], Naukova dumka, Kiev, Ukraine. (In Russ.)

2. Shamsul Huq, A.K.M. and Rosenberg, A.J.J. (1964) "Electrochemical Behavior of Nickel Compounds: I. The Hydrogen Evolution Reaction on $\mathrm{NiSi}, \mathrm{NiAs}, \mathrm{NiSb}, \mathrm{NiS}, \mathrm{NiTe}_{2}$, and Their Constituent Elements", Journal of the Electrochemical Society, vol. 111, no. 3, pp. 270-278.

3. Vijh, A.K., Belanger, G.and Jacques, R. (1990)“"Electrochemical activity of silicides of some transition metals for the hydrogen evolution reaction in acidic solutions", International Journal of Hydrogen Energy, vol. 15, no. 11, pp. 789-794.

4. Vijh, A.K., Belanger, G. and Jacques, R. (1992) "Electrolysis of water on silicides of some transition metals in alkaline solutions", International Journal of Hydrogen Energy, vol. 15 , no. 7, pp. 479-483.

5. Shein, A.B., Kichigin, V.I., Konyk, M., Romaka, L. and Stadnyk, Yu. (2013) "Study of the kinetics and mechanism of the hydrogen evolu- 
tion reaction on $\mathrm{CeMe}_{2} \mathrm{Ge}_{2}$ electrodes $(\mathrm{Me}=$ $\mathrm{Fe}, \mathrm{Co}, \mathrm{Ni})$ ", Chemistry of Metals and Alloys, vol. 6, no. 3-4, pp. 113-120.

6. Meyer, S., Nikiforov, A.V., Petrushina, I.M., Kohler, K., Christensen, E., Jensen, J.O. and Bjerrum, N.J. (2015) "Transition metal carbides ( $\mathrm{WC}, \mathrm{Mo}_{2} \mathrm{C}, \mathrm{TaC}, \mathrm{NbC}$ ) as potential electrocatalysts for the hydrogen evolution reaction (HER) at medium temperatures", International Journal of Hydrogen Energy, vol. 40, no. 7, pp. 2905-2911.

7. Safizadeh, F., Ghali, E. and Houlachi, G. (2015) "Electrocatalysis developments for hydrogen evolution reaction in alkaline solutions - A Review", International Journal of Hydrogen Energy, vol. 40, no. 1, pp. 256-274.

8. Kichigin, V.I. and Shein, A.B. (2015) "Kinetics and mechanism of hydrogen evolution reaction on cobalt silicides in alkaline solutions", Electrochimica Acta, vol. 164, pp. 260-266.

9. Kuzminykh, M.M., Panteleeva, V.V. and Shein, A.B. (2019) "Cathodic hydrogen evolution on iron disilicide. I. Alkaline solution", Izvestiya vuzov. Khimiya $i$ khimicheskaya tekhnologiya, vol. 62, no. 1, pp. 38-45. (In Russ.)

10. Kuzminykh, M.M., Panteleeva, V.V. and Shein, A.B. (2019) "Cathodic hydrogen evolu-

\section{Об авторах}

Валерия Витальевна Третьякова, магистрант, кафедра физической химии Пермский государственный национальный исследовательский университет 614990, г. Пермь, ул. Букирева, 15. https://orcid.org/0000-0002-7629-9307 lera.karaxina@yandex.ru

Анна Евгеньевна Пономарева, магистрант, кафедра физической химии Пермский государственный национальный исследовательский университет 614990, г. Пермь, ул. Букирева, 15. https://orcid.org/0000-0001-5768-435X an.pon@mail.ru tion on iron disilicide. I. Acidic solution", Izvestiya vuzov. Khimiya i khimicheskaya tekhnologiya, vol. 62 , no. 2, pp. 59-64. (In Russ.)

11. Theerthagiri, J., Lee, S.J., Murthy, A.P., Madhavan, J. and Choi, M.Y. (2020) "Fundamental aspects and recent advances in transition metal nitrides as electrocatalysts for hydrogen evolution reaction: A review", Current Opinion in Solid State and Materials Science, vol. 24, no. 1, p. 100805.

12. Gel'd, P.V. and Sidorenko, F.A. (1981) Silitsidy perekhodnykh metallov chetvertogo perioda [Silisides of transition metals of the fourth period], Metallurgiya, Moskow, Russia. (In Russ.).

13. Sukhotin, A.M. (ed.) (1981) Spravochnik po elektrokhimii [Reference book on an electrochemistry], Khimiya, Leningrad, Russia. (In Russ.).

14. Myurarka, M. (1986), Silitsidy dlya SBIS [Silicides for SBIS], Mir, Moscow, Russia. (In Russ.)

15.Zhang, X.G. (2001), Electrochemistry of silicon and its oxide, Kluwer Academic/Plenum Publ., New York, USA.

\footnotetext{
About the authors

ValeriaV. Tretyakova, Masterstudent, DepartmentofPhysicalChemistry, PermStateUniversity, 15,Bukirevstr., Perm, 614990, Russia. https://orcid.org/0000-0002-7629-9307 lera.karaxina@yandex.ru
}

Anna E. Ponomareva, Masterstudent, DepartmentofPhysicalChemistry, PermStateUniversity, 15,Bukirevstr., Perm, 614990, Russia. https://orcid.org/0000-0001-5768-435X an.pon@mail.ru 
Виктория Вячеславовна Пантелеева, кандидат химических наук, доцент кафедры физической химии Пермский государственный национальный исследовательский университет 614990, г. Пермь, ул. Букирева, 15. https://orcid.org/0000-0002-1506-6665 vikpant@mail.ru

Анатолий Борисович Шеин, доктор химических наук, профессор, профессор кафедры физической химии Пермский государственный национальный исследовательский университет 614990, г. Пермь, ул. Букирева, 15. https://orcid.org/0000-0002-2102-0436 ashein@psu.ru
Viktoria V. Panteleeva,

Candidate of Chemical Sciences,

Associate Professor, Department of Physical Chemistry,

Perm State University,

15,Bukirevstr., Perm, 614990, Russia. https://orcid.org/0000-0002-1506-6665 vikpant@mail.ru

Anatoly B. Shein, Doctor of Chemical Sciences, Professor, Professor of the Department of Physical Chemistry,

Perm State University, 15,Bukirevstr., Perm, 614990, Russia. https://orcid.org/0000-0002-2102-0436 ashein@psu.ru

\section{Информация для цитирования:}

Третьякова В.В., Пономарева А.Е., Пантелеева В.В., Шеин А.Б.Состав, структура и электрохимическая активность силицида титана в реакции выделения водорода// Вестник Пермского университета. Серия «Химия». 2021. Т. 11, вып. 4. С. 263-270. DOI: 10.17072/2223-1838-2021-4-263270.

Tretyakova V.V., Ponomareva A.E., Panteleeva V.V., Shein A.B. Composition, structure and electrochemical activity of titanium silicide in hydrogen evolution reaction, Bulletin of Perm University. Chemistry, 2021, vol. 11, no. 4, pp. 263-270. (In Russ.). DOI: 10.17072/2223-1838-2021-4-263-270. 